\title{
Protée
}

\section{Microlecture et macro-enjeux de la narration graphique. L'arpentage de Jan Baetens}

\section{Philippe Sohet}

Volume 28, numéro 1, 2000

Variations sur l'origine

URI : https://id.erudit.org/iderudit/030587ar

DOI : https://doi.org/10.7202/030587ar

Aller au sommaire du numéro

\section{Éditeur(s)}

Département des arts et lettres - Université du Québec à Chicoutimi

ISSN

0300-3523 (imprimé)

1708-2307 (numérique)

Découvrir la revue

Citer cet article

Sohet, P. (2000). Microlecture et macro-enjeux de la narration graphique. L'arpentage de Jan Baetens. Protée, 28(1), 107-112.

https://doi.org/10.7202/030587ar

\section{Résumé de l'article}

Dans le domaine de la narration graphique comme ailleurs, la crise généralisée des pratiques culturelles aura souvent stimulé le redéploiement de l'appareil théorique. Depuis quelques années, les travaux de Jan Baetens s'attachent notamment à décrire la complexité du dispositif propre aux récits en images. Privilégiant la méthode des microlectures, son dernier ouvrage, Formes et politique de la bande dessinée, analyse finement certains des paramètres expressifs (couleur, dynamique icono-linguistique, enjeu narratif) tout en s'interrogeant sur les conditions de production et la mise en circulation des oeuvres. Au-delà du domaine de la narration graphique, les perspectives évoquées constituent un outil précieux pour revoir le champ culturel dans son ensemble. 


\title{
MICRO LECTURESET MACRO-ENJEUX DE LA NARRATION GRAPHIQUE
}

\author{
L'ARPENTAGE DE JAN BAETENS
}

PHILIPPE SOHET

La crise généralisée des pratiques culturelles qui caractérise la dernière décennie du siècle appelle une réflexion de fond sur leurs modes comme sur leurs enjeux. Le domaine dela bande dessinée nefut guère épargnépar les turbulences decemouvement deressac et de redéploiement. La fermeture de «vénérables» institutions éditoriales, la politique hégémonique des «séries», le repli frileux de la production sur un lectorat restreint (largement enfantin) allaient de pair avec l'émergence d'une véritable nébuleuse de maisons de production indépendantes et la richesse détonante de leurs créations. Parallèlement, la difficultéà se doter d'un appareil critique et théorique de qualité, qui a longtemps prévalu, semble céder le pas à une nouvelle vitalité de la réflexion. C'est incontestablement de cette mouvance que relève le dernier ouvrage de Jan Baetens ${ }^{1}$.

D ans la ligne des précédentstravaux de Baetens ${ }^{2}$, Formes et politique de la bande dessinée s'impose comme un essai important dont il faut souligner avec force l'envergure des perspectives, l'acuité de la réflexion et la finesse des analyses. Intimement convaincu que «l'interaction des œuvres et de leur commentaire est indispensable à la découverte de nouvellesformes d'écritureet de lecture» (p. 2), I'auteur nous propose ici une série d'études qui relisent et font dialoguer des œuvres d'horizons, d'époqueset deprojetsvariés. H ergé et $\mathrm{H}$ erriman voisinent $\mathrm{C}$ astells ou Escher; Pratt et Swarte rencontrent $V$ andersteen alors que Breccia y annonce les productions du groupe Fréon!

La perspective privilégiée est celle des «microlectures»: analyses subtiles de certains détail s formels visant à mettreà jour les paramètres expressifs et leur interaction dans la production du récit. Contournant la tentation d'une fuite en avant formaliste, la réflexion se confronte sans arrêt aux conditions de production et de réception des œuvres, aux effets de leur mise en circulation. Pour ce faire, les analyses convoquent régulièrement les travaux les plus féconds sur la question (G roensteen, Berger, G enette, Aumont, Ricardou, $M$ arion, etc.).

La prédilection pour l'optique des microlectures n'est pas sans incidences. 0 utre la grande difficulté à rendre compte ici du degré de minutie des analyses sans en trahir toute la finesse ${ }^{3}$, ceparti prisne permet pas deviser la reconstruction synthétique ou exhaustive du système de la bande dessinée. U ne première perspective synthétique était déjà dével oppée dans Pour unelecturemodernedela bandedessinée dont le présent ouvrage vient illustrer et compléter les propositions. $N$ otons cependant que l'analyse des mécanismes dans ces opérations de sens dépasse toujours le cadre étroit del'exempleponctuel et débouched'emblée sur desperspectives réflexives d'envergure où pratiques formelles et enjeux sociaux ne sont jamais dissociés.

C ette dynamique se reflète dans les quatre thématiques regroupant les études de ce volume. Les trois premières concernent divers aspects formels (le rôle de la couleur, les rapports entre texte et image, le récit et la forme) alors que la dernièreévaluel'impact des trois perspectives précédentes sur une politique dela forme.

\section{Le paramètre chromatique}

La couleur demeure sans doutel'un des aspects les moins étudiés du dispositif expressif de la bande dessinée. Pourtant, les trois études que lui consacre J an Baetens le démontrent, le phénomène est loin de se limiter au simple coloriage dans une perspective d'embellissement ou d'un surcroît de réalisme.

Pour s'en convaincreil n'est que de relirecertainstravaux d'H ergé. L'utilisation dela couleur sevoit habilement insérée au sein des stratégies narratives déployées. Elle appuie, par exemple, la dynamique d'alternance entre les seconds plans 
de «décor» (détaillés) ou de «fond» (neutres) qui constituait I'une des bases de la rythmique narrative d'H ergé, supportée par d'autres variations des paramètres expressifs:

Chaquefoisqueledécor semétamorphoseen fond, $H$ ergéchange de point de vue comme de cadrage: le point de vue devient frontal [... ] et lespersonnagessont montrésla plupart du temps en plan rapproché. (p.8)

B risant une perspective strictement référentielle, des coloris distincts viendront ponctuer fond et décor. Cettedynamique apparaît avec le plus d'éclat dans la célèbre séquence du combat entre Rackham le R ougeet le $C$ hevalier F rançois de $\mathrm{H}$ adoque (qui, est-il besoin de la rappeler, se joue à deux niveaux narratifs: celui du combat lui-même et celui du récit véhément que nous en fait le capitaine dans son appartement). Or, non seulement l'alternance entre fond et décor soutient ce double niveau (la narration au passé privilégiant le décor, la narration au présent le fond), mais le lecteur assistera à une variation (exceptionnelle au sein de l'œuvre d'H ergé) dans le coloris du fond des scènes del'appartement selon l'intensité des phases du combat de l'ancêtre.

Cet exempled'utilisation structurée de la couleur au sein des dispositifs expressifs dela bande dessinéen'est pas unique, ni même premier. Lorsqu'en 1935, sous la pression commerciale des éditeurs, G eorge H erriman se voit pressé d'introduire la couleur dans ses célèbres «strips» de Krazy Kat, c'est à une réorganisation complète des paramètres que le lecteur assistera:

[... ] la division paginale de K razy Kat [... ] passe en peu de tempsdela formulela plus conventionnellequi soit à une série de modèles d'une li berté presque anarchique. (p.17)

Cette effervescence, note Baetens, est le résultat d'un déplacement, d'un rééquilibrage dû aux effets de l'introduction dela couleur. En effet, la fantaisieuniquedel'univers de Krazy Kat reposait en partie sur la dynamique débridée entre l'avant et l'arrière-plan où les détails se modifiaient à profusion. $0 r$, avec le recours à la couleur, les plages d'aplats qu'elle dispose atténuent la reproduction de détails très fins et restreignent par le fait même la portée significative du décor. C ette effervescencesembledonc bien êtreune «mesure pour compenser hors vignette ce qui se perd à l'intérieur de la vignette».

Plus récemment, certaines pratiques de la «couleur directe» (appliquée à même la planche originale, non plus à partir d'un calque) et la contestation des surfaces closes qu'imposait le graphisme (longtemps hégémonique) de la «ligne claire» ont rapproché certaines manifestations de la bande dessinée des pratiques picturales et expressives de la peinture, induisant du même coup de nouvelles approches du phénomène. Certains récits de $C$ astells sont habilement travaillés par ces «liens controversés entre bande dessinée et peinture» (p. 28). L'analyse minutieuse d' El Sr. Go yla joven Anteas révèle l'allégorie qui traverse cette rencontre entre M onsieur $G o$ et Antée, entre la bande dessinée et la peinture, et comment, au terme de l'exposition et de la confrontation de leurs dispositifs, la narration graphique peut prendre habilement appui sur certains constituants de la peinture, notamment son utilisation pensée du chromatisme ( puisque dans ce récit «les rapports entre cases sont souvent plus chromatiques que directement narratifs», p. 33).

\section{La lettre et l'icône}

Interrogeant ensuite les interactions del'imageet du texte dans la bande dessinée, Jan Baetens nous oblige encore à reconsidérer certaines évidences trop aisément acquises et à poursuivre la réflexion dans de nouvelles directions. Par essence, la bande dessinée est un médium polysémiotique. L'histoire précise des modalités d'articulation des codes iconiques et linguistiques reste pourtant encore largement à écrire. $\mathrm{D}$ ans le contexte de production propre à la bande dessinée et face aux préjugés qui y restèrent longtemps rattachés, la non-redondance des deux registres mit du temps à s'affirmer. Demême, malgréles coups deboutoir decertaines productions contemporaines innovatrices, le primat d'un code (linguistique) sur l'autre (iconique) resteencore souvent une donnée communément acceptée. T rois études permettront à l'auteur de raffiner les perceptions des mécanismes expressifs en ce domaine et d'en mesurer les enjeux réels.

D ans un premier temps, il nous rappelle qu'avant d'être lettre ou icône, la bande dessinée est essentiellement graphique. Elle ne peut, de ce fait, échapper au «style» d'un auteur dont elle sera toujours la trace. S'appuyant sur les travaux de Philippe $\mathrm{M}$ arion 4 , il plaide à son tour pour I'introduction du concept de graphiation:

[... ] la graphiation est donc fondamentalement autoréférentielle. Avant de contribuer à la figuration, elleest automonstration, dansla traced'uneidentitégraphiqueperceptible dans la spécificitésubjective d'une empreinte. 5

D ans le processus de l'énonciation narrative, la graphiation constituerait ainsi, aux yeux du lecteur, une couche 
«antérieure» à la narration et même à la monstration. La présence de cette graphiation varie évidemment en forme et en intensité. Elle se fait plus forte dans les effets d'esquisses et plus ténue lorsque le dessin tend à une «évocation transparente de ce qui se voit figuré».

$M$ ais, avertit Baetens, il ne faudrait pas trop rapidement rabattrela notion degraphiation à celled'un graphiateur, car il ne s'agit pas tant d'une personne que d'une instance. En effet, d'une part les contingences de la production peuvent multiplier les interventions d'agents divers dans la mise en traits (le dessinateur mais aussi, l'encreur, le «lettreur » et le coloriste, par exemple) et, d'autre part, le travail graphique est loin d'être «naturel», il relève d'un actesocialisérépondant à des codes: contraintes d'écoles et d'époques.

[... ] chaquestyle, loin deseréduireà la manifestation d'un moi, signifie d'abord un choix dans l'éventail des syles disponibles à un moment donné, et une préférence quant à la manière dont cette parole chercheà être compriseet interprécée. (p. 42)

La prise en compte de cette dimension, généralement ignorée dans la maj orité des théories dela narration graphique, ouvre des perspectives nouvelles mettant l'accent sur la puissance narrative d'éléments graphiés propres au médium.

S'éloignant quelque peu de la bande dessinée, Jan Baetens s'appuie ensuite sur une gravure de M .C. Escher (Le Roulenboule, 1951) pour démontrer combien, face au poids des traditions, s'opposent certaines productions dont l'audace offre une réelle ouverture de perspective et qui, dès lors, prennent valeur emblématique. Ainsi, régulièrement,

[... ] l'imageest étudiéecommeun signe spatial dont les parties s'offrent simultanément à la vue, là où rivéeà la ligne, l'éciture se prête à une lecture essentiellement temporelle. (p. 47)

O r la gravure en question fait cohabiter signes scripturaux et analogiques mais dans une articulation qui tente précisément «un échange généralisé de leurs traits spécifiques» (p. 48). D ans un détournement dela planched'encyclopédie, la gravureprésenteun animal imaginaire, sorte de mille-pattes se déplaçant par «auto-enroulement» tandis qu'un long commentaire descriptif nous en rapporte les mœurs. $M$ ais, contrairement à la coutume, la cohabitation du texte et de l'image ne se réduit pas à une simple juxtaposition, voire à l'incrustation de celleci dans celui-là. L'agencement, ici, stigmatise lestraits spécifiques des matériaux pour les contourner. La partieimagées'étal esur unelaize diagonale reliant le point de départ du parcours de la lectureà son point d'achèvement et, en présentant les étapes d'enroulement de l'animal, se «séquentialise» comme toute phrase. À l'inverse, le texte, clôturé aux deux aires que sépare ce trajet, perd de sa linéarité pour se donner d'abord comme figures, surfaces triangulaires dont la régularité interlignaire est ajustée au rythme d'enroulement de la figure. Cette réalisation du maitre hollandais, où l'image semble se linéariser et l'écrit prendre figurespatiale, vient donc opportunément rappeler la richesse potentielle des territoires sémiotiques qu'il resteà la narration graphique à explorer.

D es territoires que même $\mathrm{H}$ ergé, derrière le classicisme de son style, n'a pas hésité à effleurer. Avec discrétion mais combien d'efficacité. Poursuivant ici la démarchequi fondait déjà son audacieux «H ergéécrivain », Baetensnous démontre (et démonte) l'étonnante lucidité hergéenne aux registres signifiants. Michel Serres fut l'un des premiers à souligner l'existence d'une théorie de la communication à tirer des lapsus communicationnels dans I'univers de T intin ${ }^{6}$. D ans la même perspective, s'attachant aux pirouettes verbales de Tournesol, J an Baetens y met à jour l'étonnant dialogue des ressources verbales et visuelles. Ainsi, comment expliquer quela surditédu personnage se doubletoujours d'un trouble visuel? C urieusement, si l'acuitévisuelledeT ournesol semble intactetant qu'aucuneparolenes'échange, dès que se déploie le dialogue, une curieuse cécité surgit chez le professeur qui «réussit à renverser la signification des physionomies les plus comminatoires» (p.59) par un incroyable effet de contamination du linguistique sur le visuel. Cet entrelacs de deux matières expressives se retrouve encoreà divers niveaux dans I'univers hergéen, notamment quand l'image se donne littéral ement à «lire», Q uele récit remplace les réserves deboisson du capitaine, ce sera bien entendu pour y substituer une «boîte de biscuits» dont la composition phonique ne peut que redoubler I'opération en rappelant la bouteille de whisky brisée, gisant à côté de la chal oupe:

D 'un côté, le début du mot boî-te est identiqueà celui de la famille lexicale du terme boi-sson. D e l'autre, le noyau du vocableb-iscui-tsreprend demanièrechiasmatiquelematériau phonique et consonantique intégral de W hisky. Q ui plus est, la syllabeclausulairedebis-cuitsn'est passansparentéprofonde avec la cuite dont les symptômes pullulent, comme le profond sommeil de T ournesol ou la saleté des lieux. (p.63)

\section{Les paramètres au service du récit}

U netroisième section du volumeanalyse la contribution de certains détails formels à la construction du récit. D issé 
quant une ancienne production du flamand $\mathrm{V}$ andersteen 7 , l'auteur cherche à identifier certains des soubassements formels du projet narratif et, dans ce cas précis, à «saisir les relations inextricables qui se nouent entre trois éléments fondateurs: langue, rythme et récit».

L'exemple retenu se révèle particulièrement opportun puisquel'effervescence débridéedu ton et du style (qui échappaient encore aux futurs diktats du genre qu'imposera sous peu l'assagissement institutionnel du médium) laisse apparaitre avec plus d'acuitél'organisation formelle des procédés en place. Certesla vitalitédu récit tient en partieàla structure feuilletonesque (que favorisait son rythme de publication d'origine), mais également à l'habile articulation des paramètres thématiques, iconiques et langagiers pour créer la vivacitédu rythme. Leprétextelui-même(une course-poursuite) se concentre essentiellement sur l'action: toute vignette à portée quelque peu descriptive, toute motivation psychologisante qui risqueraient de freiner la lecture semblent avoir été écartées, c'est bien le mouvement lui-même qui devient I'objet de cet emballement narratif. Avec finesse, Baetens décrit les «paramètres visuels qui aident à entretenir lerythme à l'intérieur de la case et dans les rapports d'une vignette à l'autre» (p. 98). La logiquequi prévaut au découpage s'avère particulièrement efficace: chaque case présente une action quasiment distincte, les personnages n'apparaissent jamais en état d'immobilité, et leurs actionsnesont guèresynchrones ou comparables, multipliées souvent par un recours systé matique aux possibilités qu'offre lehors champ. D emultiples inversions paramétriques viennent en dosser et poursuivrece mouvement général : ainsi la composition des vignettes (qui alterne l'orientation des axes dominants et «chaloupe» le parcours del'œil) est conjuguée avec des variations aussi systé matiques dans la distance, le point de vue ou le cadrage. La dimension langagière n'est pas en reste et $V$ andersteen aura su jouer au mieux de «toutes les possibilités rythmiques offertes par le texte» (p.104). N on seulement par l'elliptique des dialogues, mais également par la «répartition concertée des masses verbales» créant une sorte de ping-pong visuel entre les cases, sans oublier le recours au dialecte «qui le rapproche de l'expérience carnavalesque» et aux calembours qui semblent à l'origine de certains comportements faisant ainsi du récit «la conséquence de cettemanipulation ludique des mots pour le dire» (p. 105).

La pertinence des observations avancées par Baetens ne peut que stimuler son lecteur à poursuivre la réflexion en se demandant, par exemple, si la richesse del'exemple retenu ne tient pas autant à ce qu'il ne montre pas qu'à ce qu'il expose. $C$ ar leprojet narratif de $V$ andersteen dans cette aventuren'est pas sans se rapprocher d'autres projets, notamment celui de Forton dans certains épisodes des célèbres Pieds N ickelés avec leur gouaillerie, leur sens de la dérision et la cascade désordonnée d'événements. C eque révèle précisément le travail de $\checkmark$ andersteen, c'est bien l'écart structurel qui distingueles deux réalisations éloignées de quelques décennies. II faudra relire les albums de Forton à la lumière des remarques de l'auteur pour mieux mesurer la maîtrise des paramètres qui s'affirme d'un dessinateur à un autre: là où l'expression graphique de Forton n'offrait qu'unepal etteexpressived'une granderigidité (tant dans la hauteur des cases que dans le choix du cadrage, de la perspective et de la position), le «strip» de V andersteen modulefrénétiquement ces mêmesparamètres. Cequi s'exhibe ainsi, c'est la mise en place d'un dispositif denarration original qui aura (enfin?) permisl'élargissement du récit en images hors dela domination du matériel scripturaire, au serviced'un projet narratif propre: celui de la bande dessinée.

$M$ ais le récit peut reposer sur diverses configurations. L'analyse d'une pochette de disque révélera la virtuosité et les artifices par lesquels le dessinateur J oost Swarte réussit à condenser le «déroulement narratif » en une seule scène. Sur la vignette, une jeunefille, devant une affiched'un musicien, gonfle sa robe au vent par un gracieux mouvement dansé. Les yeux ébahis d'un cycliste qui a mis pied à terre pour l'occasion et le carambolage de plusieurs automobiles en arrière-plan nous laissent deviner ce que, par la miseen scène, le mouvement de jupe soustrayait à nos yeux de spectateurs. Sur cette vignette, succession des plans et juxtaposition des niveaux entretiennent d'étranges échos sémantiques et syntaxiques. Les événements au loin semblent répondre aux éléments de premier plan et cette danse prendre son origine danslemotif pourtant «affiché» decemusicien (présentéen pleine action). La construction formelle (la position et la composition de l'affiche, le dialogue de la plongée et de la contre plongée) crée une métal epse optique qui aboutit «à la fusion desniveaux fictionnels qui cohabitent sur la feuille» (p.73). D ans cette mise en scène affolante, Swarte prend donc un mal in plaisir à exploiter deux des constituantsfondamentaux du système perspectif traditionnel : la ségrégation des plans (qui réduit l'étendueà quelques plans sélectionnés) et la veduta (ouverture d'un plan sur un autre) en les détournant au seul profit du projet narratif.

$D$ ansun autre chapitre, relativement dense et nettement plus audacieux, Baetens souscrit aux perspectives de la 
textique et des travaux de Ricardou élargissant la notion de récit du strict niveau diégétique au «déchiffrement vectorisé de l'objet à lire». II se propose alors d'explorer certaines frontières du projet narratif en analysant ce

[... ] no man's land entre l'infranarratif de certaines compositions paginales entièrement tournées du côté du tabulaireet ledémarrage récitatif orientéverslelinéai re. (p.91)

Appuyant sa démonstration sur l'analyse d'une planchecouverture ( $T$ ango deH ugo Pratt) où le regroupement d'une série de vignettes ne semble, de fait, guère être régi par un projet séquentiel (syntagmatique), mais offrir davantageune série de vues partielles d'un coupledansant. Pourtant I'organisation des vignettes, tant dans leur composition que dans leurs agencements multiples, lui paraît révéler des «mouvements» formels: établissement, ruptureet rétablissement de principes, ou encore parallélismes et échos entre divers niveaux structuraux. D ans une telle optique, I'analyse vise à rendre compte des

[... ] aventures de la forme qui, de manière indépendante ou en dialogue avec les événements racontés, s'avère à même d'enclencher une lecture narrative dirigée vers ce qu'on a pu appeler le «récit des paramètres». (p. 77)

\section{Une politique de la forme}

La dernière section de l'ouvrage réinscrit l'ensemble des réflexions qui la précèdent dans une perspective globale, évaluant les enjeux de ce qui se donnerait alors comme une «politiquedela forme». T rop souvent, l'analyse du politique au sein des productions culturelles se voit restreinte à l'évaluation axiologique de leurs contenus, alorsqu'il est possible del'aborder égal ement «commeune stratégie destinée à ressortir des effets à l'intérieur d'un contexte déterminé» (p.137). D ans la ligne des travaux de Ricardou, il s'agit davantage dejauger la capacité de certaines œuvres ou courants à

[... ] souligner structuralement ces opérationset paramètresque le régime représentatif, avec sa primauté du message à communiquer, occulte en partie ou totalement. (p.110)

Avoir choisi comme premier exemple Ché, l'album issu de la collaboration des frères Breccia et d'O esterheld n'est pas sans poser quelques défis. $L$ '«appartenance sans nulle réserve au sous-genre honni de la propagande politique» (p.111) ne le disqualifiet-elle pas d'emblée? O r, l'analyse de Baetens le montre éloquemment, la force de l'ouvrage, son pouvoir mobilisateur réside peut-être moins dans son projet hagiographique que dans sa mise à nu de certains mécanismes du mirage représentatif. Le dispositif narratif de l'album s'articule autour de deux types de séquences relatant l'une le dernier combat en Bolivie, l'autre des élémentsemblématiques deson existence. Procédérelativement classique, même pour sa date de création (les années 60), n'était-ce l'opposition systématique des paramètres qui les constituent. Q uece soit au niveau de la voix (parcimonieuse ou diserte), de la vitesse narrative (continue ou discontinue) ou de la composition graphique (focalisée sur un objet, un personnage ou diffractée en unemultitude de plans), les deux niveaux n'auront de cesse de se révéler mutuellement leurs artifices. Au niveau expressif, I'utilisation singulière du noir et du blanc vivifie "cette tension entre deux postures représentative et métareprésentative» (p.118). Car leur organisation nese sent plus liéeà une motivation strictement réaliste: leur contrastene marquant plus nécessairement une opposition présente dans le référent, elle se donne d'autant mieux à lire comme aspect matériel de l'expression. II en va du même projet lorsqu'une utilisation contrastée des plans exacerbe la bidimensionnalitédu support et que le recoursà quel ques formes simples, ambiguës et polyvalentes hypostasie la force de toute interprétation lectorielle. Ces manœuvres n'empêcheront évidemment pas d'y lireun projet idéologique en ce qu'elles visent souvent à permettre le passage du biographique à l'hagiographique mais elles «font de Chéun de ces ouvrages où le méticuleux travail sur la forme épaule la transmission d'un message univoque» (p. 114).

Une telle préoccupation d'inscrire une réflexion sur la forme et le dispositif représentatif au sein même de l'œuvre semble bien caractériser unepart importantede la production récente de jeunes auteurs regroupés dans la nébuleuse du réseau d'Autarcic $\mathrm{C}_{\text {omix }}{ }^{8}$. S'attachant plus particulièrement aux travaux du groupe belge $F$ réon et à leur revue $F$ rigobox, l'auteur analyse comment «l'indignation à fleur de peau, I'urgence politique, le besoin d'en découdre avec la société» (p.127) ne peuvent y être séparés de l'examen des traits formels de leurs travaux, de leurs efforts pour se doter de nouvelles expressions.

Rejoignant les critiques les plus acérées du régime médiatique, c'est bien àuneversion contemporaine de la querelle des iconoclastes qu'ils nous sensibilisent, dénonçant avec vigueur la profusion et le brouillage des images où le réel tend de plus en plus à se confondre avec ses représentations et où «l'ennemi, c'est l'excès d'images et de texte qui coupe les ponts entre le sujet et le réel » (p. 140). O n en comprend 
d'autant mieux la méfiance affichée par le groupe à l'égard de cette frénésie postmoderne de la «poétique de la citation tous azimuts» et à ses «collages irréfléchis» (p.122), tout commeleur volonté d'évitement de références à des modèles stylistiques dominants (la fameuse «ligne claire»). M ais le projet n'en resterait qu'au stade de la crispation stériles'il ne débouchait pas sur une production «où les anciennes contraintes ne représentent plus I'horizon englobant, mais [... ] un point de départ» (p. 146). O r, à considérer les productions du groupe Fréon, il semble possible de synthétiser l'originalité de leurs efforts dans leur volonté de dépasser certaines clôtures. Jan Baetens identifie quatre traits fondamentaux de cette production qui se donnent comme autant de revendications: dépassement de l'«homogénéité de matière comme de style» (par la coprésence de multiples classes d'images venant romprel'illusion représentative mise en place), dépassement de la tutelle des genres (le refus de restreindre le champ de la bande dessinée à un ou quelques genres dominants), dépassement du récit (avec unesensibilité au récit des formes où «le lecteur se voit incité à lui-même retracer le vagabondage et les métamorphoses d'une tache, les places variables d'un trait, les tremblés ou dilutions d'une égratignure», p.132), dépassement même du livre (avec l'exploration dela mise en scène[narrative] in situ de certaines de leurs expositions ${ }^{9}$ ). Et l'auteur de conclure:

L'apport politique majeur de Frigobox réside peut-être à ce niveau-là. Le travail sur la transgression, imperfections et réussitesconfondues, montrequ'il ya toujoursloisir dechanger les règles du jeu. (p.147)

\section{L'arpentage de la narration graphique}

D epuis des années, Jan Baetens arpente le champ des récits en images. Les analyses qu'il nous propose ici sont autant d'incursions dans un territoire dont la richesse potentielle demeure sous-estimée. Étoffées, fécondes, voire provocantes ${ }^{10}$, ses microlectures balisent peu à peu la complexité du dispositif propre à la narration graphique. Q ue l'on ne s'y trompe pas cependant: si les analyses déployées dans ce volume se concentrent davantage autour du domaine de la bande dessinée, les réflexions qu'elles engendrent dépassent largement le secteur et constituent un levier précieux pour questionner lechamp culturel danstoute son extension. Elles s'offrent comme un irremplaçable appel àl'intelligence dela lecture. À ceseul titre, Formeset politique dela bandedessinée s'impose comme un détour obligé.

\section{N OTES}

1. J. Baetens, Formeset politiquedela bandedessinée, Leuven (Belgique), Éd. Peeters V rin, coll. «Accents», 1998, 151 p., 7 illus.. Aux côtés de l'ouvrage deBaetens, il serait injustedenepas mentionner, entreautres, Forginga new medium de C. Dierick et P. Lefèvre (VUB Press, 1998) ainsi que la publication du Systèmedela bandedessinéede T . G roensteen, (Paris, P.U .F., 1999). Le secteur des revues n'est pas en reste avec $9^{\circ}$ art du CN BD I (Angoulême) ou le tout récent International J ournal of C omic Art (c/0 J ohn Lent, 669 Ferme Bvd, D rexel H ill, PA 10926 USA).

2. Enseignant aux universités de Leuven et de M aastricht, J. Baetens a publié de nombreux ouvrages dans le champ des «cultural studies» et notamment de la bande dessinée. Relevons, parmi les plus récents, $H$ ergé écrivain (Labor, 1989), Pour unelecturemodernedela bandedessinée (avec $P$. Lefèvre, Centre belge de la bande dessinée, 1992), D u Photo-roman (Les impressions nouvelles, 1993), Le Réseau Peeters (Rodopi, 1995).

3. D ont lelecteur aura déjà pu serendre compteen prenant connaissance de l'étude que publiait dernièrement ). Baetens («D éjà fini ! Q uel dommage! ». M icrolecture d'un incipit: Le Réseau M alou) dans Recherches en communication (n08, 1997, Louvain-la-N euve, p. 87-102.)

4. Tracesen cases $T$ ravail graphique, figuration narrativeet participation du lecteur (Essai sur la bandedessinée), Louvain-la-N euve, Éd. Academia, 1993.

5. P. M arion, cité par J. Baetens, p. 37.

6. «Lesbijouxdistraitsou lacantatricesauve», dans H ermès I . L'Interférence, Paris, Éd. de M inuit, 1972.

7. Cerécit, publiéentre 1948 et 1950, nepossédait pas deversion française au moment dela rédaction del'analyse de J. Baetensqui lenommedansson texteL' $H$ urluberlu calibré. D epuis, unetraduction est disponiblesousletitre Les Q uipropos d'un quidam, Éd. Standaard, 1995.

8. Label qui regroupe les travaux de diverses petites maisons d'édition indépendantes telles que Amok, La Cinquième couche, Fréon, Ego commeX, L'Association, etc.

9. Invitédanslecadre dela participation belgeau festival d'Angoulême99, le groupe Fréon disposa, dans la grande salle du conseil municipal, une architecture remarquable dont les six pièces proposaient des installations narratives distinctes.

10. Ainsi le choix d'élargir le concept de récit jusqu'à la frontière du «récit des paramètres» n'est pas sans poser des questions sur la nature même du narratif et, du point de vue lectoriel, sur la façon dont l'analyse se démarque deladescription articuléedu «texte»proposé. L'auteur avait déjàhabilement soutenu dans un de ses ouvrages précédents ( $\mathrm{D}$ u Photo-roman, op. cit.) cette thèse qui relève d'une discussion qui reste à poursuivre mais dont l'enjeu dépasse les propos du volume, a fortiori de ces lignes. 